\title{
The role of genistein in experimental hepatic ischemia- reperfusion model in rats
}

\author{
Akinci $\mathrm{O}^{1}$, Durgun $\mathrm{V}^{2}$, Kepil $\mathrm{N}^{3}$, Ergun $\mathrm{S}^{4}$, Tosun $\mathrm{Y}^{5}$, Goksoy $\mathrm{E}^{2}$ \\ Department of General Surgery, Hakkari State Hospital, Hakkari, Turkey. ozanakinci1987@hotmail.com
}

\begin{abstract}
Genistein is a natural compound from the class of isoflavonoids found in high concentrations in legumes and soybeans. In this experimental study; we suggest that genistein might cause favorable outcomes in the hepatic surgery because of its protective effects on hepatic ischemia-reperfusion injury (Tab. 2, Fig. 6, Ref. 28). Text in PDF www.elis.sk.

KEY WORDS: genistein, isoflavonoids,legumes, soybeans, hepatic surgery, ischemia-reperfusion injury.
\end{abstract}

\section{Introduction}

Ischemia is the lack of sufficient blood supply and other metabolites to the tissue and impairment of the removal of cellular waste products. Restoration of the blood flow in viable ischemic tissue paradoxically causes increased and accelerated tissue damage. This results in cell death in addition to irreversible damage in the tissue following ischemic period. Factors such as polymorphonuclear leukocytes, complement system, cytokines and endothelial cells, which are formed rapidly by the introduction of molecular oxygen into the cell, and free oxygen radicals, are responsible for the damage mechanism developed during the reperfusion period $(1,2)$.

Hepatic ischemia-reperfusion injury may occur after liver transplantation and liver resection, in hemorrhagic shock, severe trauma and late stage of sepsis.

Ischemia-reperfusion injury causes significant biochemical and histopathological changes in the liver. There are many interrelated complex mechanisms in the physiopathology of these alterations including the release of various inflammatory mediators such as Kupffer cell activation, tumor necrosis factor $\alpha$ (TNF- $\alpha$ ), interleukin 6 (IL-6), increased production of free oxygen radicals, slowing of the removal of these increased radicals, deterioration of the nitric oxide and endothelin balance, change in the mito-

${ }^{1}$ Department of General Surgery, Hakkari State Hospital, Hakkari, Turkey, ${ }^{2}$ Department of General Surgery, Cerrahpasa Faculty of Medicine, Istanbul University Cerrahpasa, Istanbul, Turkey, ${ }^{3}$ Department of Pathology, Cerrahpasa Faculty of Medicine, Istanbul University Cerrahpasa, Istanbul, Turkey, ${ }^{4}$ Department of General Surgery, Istanbul Avcilar Murat Koluk State Hospital, Istanbul, Turkey, and ${ }^{5}$ Department of General Surgery, Istanbul Kartal Dr. Lutfi Kirdar Training and Research Hospital, Istanbul, Turkey

Address for correspondence: O. Akinci MD, Hakkari Devlet Hastanesi, Genel Cerrahi Klinigi, Daggol Mah. Genclik Cad. No:2, Merkez, Hakkari, Turkey, Postal zip code: 30000.

Phone: +90.5377490641 , Fax: +90.4382116067 chondrial permeability and systemic polymorphonuclear leukocyte flow $(3,4,5,6)$.

Plant-derived antioxidants (e.g., phenolic acid, catechin, flavonoids, etc.) play an important role in the capture and neutralization of free oxygen radicals by their redox capacities (7). The genistein, which is well known for its antioxidant and anti-inflammatory properties, is a natural compound of isoflavonoids. Many experiments have shown that genistein is predominantly found in most soy foods, protects cells against reactive oxygen species by clearing free radicals and reducing the expression of genes associated with cellular stress (8). Previous experimental studies have reported that genistein has protective effects in the ischemia-reperfusion injury model of the kidney, the brain, and the retina $(9,10,11)$. However, the effects of genistein on liver ischemia-reperfusion injury are not known yet.

In this experimental study; we aimed to investigate the effects of genistein on the oxidant-antioxidant system and histopathology of the liver in hepatic ischemia-reperfusion injury in rats.

\section{Materials and methods}

The present study was performed at Istanbul University Aziz Sancar Experimental Medicine Research Institute after the approval of Istanbul University Animal Experiments Local Ethics Committee (approval date and number: 10.08.2017/ 303098).

In the study, 32 male Wistar Albino rats weighing 200-250 $\mathrm{g}$ were used. Rats were housed in groups of four in cages with a temperature of $21 \pm 1{ }^{\circ} \mathrm{C}$ and 12 hours of light / dark cycles, fed with standard laboratory diet and tap water ad libitum. The rats were randomized into 4 groups as follows:

Group I: Sham group: In this group, the rats underwent laparotomy without experimental hepatic ischemia-reperfusion injury and without medical treatment. Blood and liver tissue samples were taken. 
Group II: Ischemia-Reperfusion (I/R) Group: No treatment was given to the rats in this group. After laparotomy, hepatic pedicle was occluded with an atraumatic vascular clamp for 15 minutes. Blood and liver tissue samples were taken after 20 minutes of reperfusion.

Group III: Genistein group: In this group, the rats were given 1 $\mathrm{mg} / \mathrm{kg}$ of subcutaneous genistein (Sigma Aldrich, USA) injection 24 hours and an hour before the procedure. Hepatic ischemia-reperfusion model was not applied to the rats. Laparotomy was performed under general anesthesia. Blood and liver tissue samples were obtained immediately after laparotomy.

Group IV: Genistein + Ischemia-Reperfusion (I/R) Group: The rats in this group received genistein $1 \mathrm{mg} / \mathrm{kg}$ subcutaneously 24 hours and an hour before the procedure. Laparotomy was performed under general anesthesia. The hepatic pedicle was occluded with an atraumatic vascular clamp for 15 minutes. Blood and liver tissue samples were taken after 20 minutes of reperfusion.

All surgical procedures were performed using sterile surgical instruments. All surgical procedures were performed between 09:00 and 12:00, to overcome the possible effects of diurnal hormonal changes on rats. The rats were anesthetized with an intramuscular injection of $10 \mathrm{mg} / \mathrm{kg}$ xylazine (Alfazyne, $2 \%$, Alfasan, Woerden, Holland) and $80 \mathrm{mg} / \mathrm{kg}$ ketamine (Ketalar, Pfizer Pharma, GMBH Germany).

Blood samples were obtained, and the measurements of alanine aminotransferase (ALT), aspartate aminotransferase (AST), lactate dehydrogenase (LDH), TNF- $\alpha$, IL-6 were performed. Superoxide dismutase (SOD) and glutathione peroxidase (GPx) were measured in liver tissue samples.

Liver tissue samples obtained for histopathological investigation were examined for hepatocyte damage. The changes in the cell histology were assessed by a single pathologist in terms of congestion, necrosis, cytoplasmic vacuolization and eosinophilia, nuclear pyknosis and inflammatory cell density. All of these parameters were evaluated according to the pathological scoring system defined by Suzuki et al.; 0: no damage, 1: minimal damage, 2: mild damage, 3: moderate damage, 4: severely damaged (12).

\section{Statistical analyses}

Descriptive data were shown as mean \pm standard deviation and median minimum-maximum values. Kolmogorov-Smirnov test was used to evaluate the normality of distribution of the data.

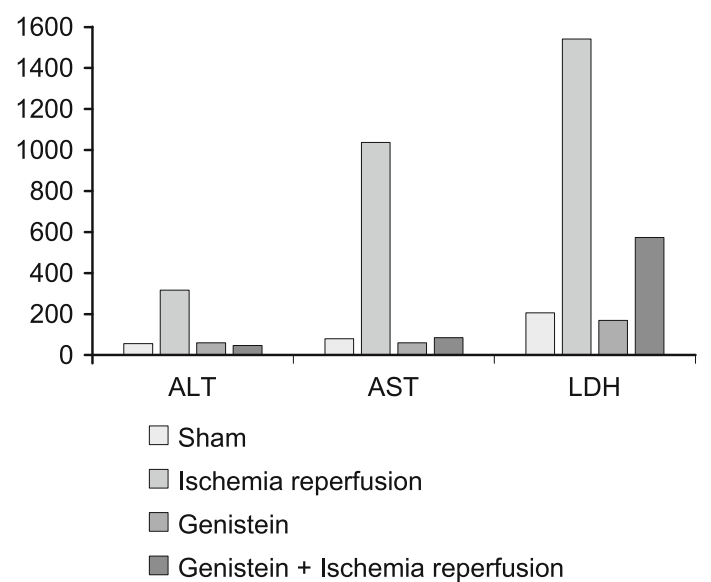

Fig. 1. The comparison of the serum ALT, AST and LDH levels of the groups.

One-way ANOVA test was used to evaluate the difference of the parameters including ALT, AST, LDH, TNF- $\alpha$, IL-6 and SOD and GPx measurements of liver homogenate. Tukey's test was used for post-hoc comparisons. Fisher's Exact test was used to compare the categorical data. A p-value of less than 0.05 was accepted to be significant for all statistical analyses. The analysis was carried out with IBM (C) SPSS software version 20.

\section{Results}

The mean ALT, AST, LDH, TNF- $\alpha$, IL-6, SOD, and GPx values of the groups are shown in Table 1.

A significant difference was found between the sham group and I/R group with respect to serum ALT, AST levels. Besides, the levels of ALT and AST were significantly lower in the genistein $+\mathrm{I} / \mathrm{R}$ group compared to the $\mathrm{I} / \mathrm{R}$ group $(\mathrm{p}<0.001)$ (Fig. 1).

The mean LDH value was highest in the I/R group, and it was significantly lower in the Genistein $+I / R$ group $(p<0.001)$.

The mean TNF- $\alpha$ level of the I/R group was higher than the other groups. The mean TNF- $\alpha$ level of the genistein I/R group was significantly lower than the I/R group $(p<0.001)$.

There was a significant difference between groups with respect to serum IL-6 levels $(\mathrm{p}<0.001)$. The mean IL-6 levels of the I/R, and the Genistein I/R groups were significantly higher than those of the sham, and genistein groups. However, the difference be-

Tab. 1. The mean ALT, AST, LDH, IL-6, TNF- $\alpha$, SOD and GPx values of the groups.

\begin{tabular}{|c|c|c|c|c|c|}
\hline & Sham $^{1}$ & ${\text { Ischemia Reperfusion }{ }^{2}}^{2}$ & Genistein $^{3}$ & Genistein+Ischemia Reperfusion $^{4}$ & $\mathrm{p}$ \\
\hline ALT & $55.4 \pm 7.2$ & $317.0 \pm 60.6$ & $59.9 \pm 16.1$ & $46.2 \pm 7.4$ & $<0.001^{\mathrm{a}}$ \\
\hline AST & $80.0 \pm 4.9$ & $1036.6 \pm 168.6$ & $60.1 \pm 15.1$ & $85.0 \pm 12.8$ & $<0.001^{\mathrm{a}}$ \\
\hline LDH & $206.4 \pm 12.2$ & $1542.0 \pm 425.3$ & $169.7 \pm 17.1$ & $573.9 \pm 196.4$ & $<0.001^{\mathrm{ab}}$ \\
\hline IL-6 & $2.3 \pm 0.9$ & $20.1 \pm 7.5$ & $8.9 \pm 4.5$ & $25.4 \pm 8.7$ & $<0.001^{\mathrm{c}}$ \\
\hline TNF- $\alpha$ & $12.2 \pm 0.5$ & $252.4 \pm 70.4$ & $17.0 \pm 4.2$ & $17.1 \pm 3.2$ & $<0.001^{\mathrm{a}}$ \\
\hline SOD & $59.1 \pm 15.7$ & $159.1 \pm 19.8$ & $121.9 \pm 14.3$ & $340.3 \pm 85.5$ & $<0.001^{\text {bd }}$ \\
\hline GPx & $30.0 \pm 2.7$ & $20.9 \pm 5.2$ & $45.3 \pm 5.7$ & $38.3 \pm 2.7$ & $<0.001^{\mathrm{e}}$ \\
\hline
\end{tabular}

Post-hoc significance: ${ }^{\mathrm{a}}$ compared to $2,{ }^{\mathrm{b}}$ compared to $4,{ }^{\mathrm{c}}$ all comparisons except $1-3$, and $2-4,{ }^{\mathrm{d}}$ compared to 1 , ${ }^{\mathrm{e}}$ All binary comparisons 


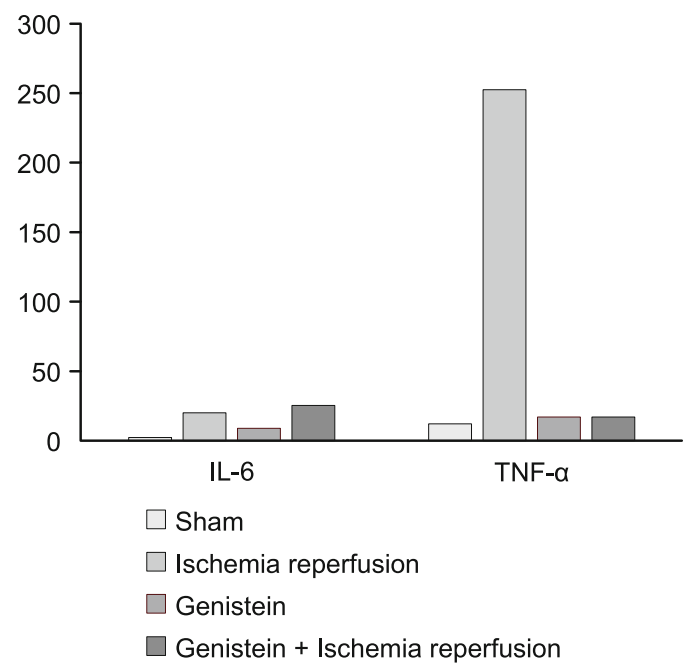

Fig. 2. The comparison of the serum IL-6 and TNF- $\alpha$ levels of the groups.

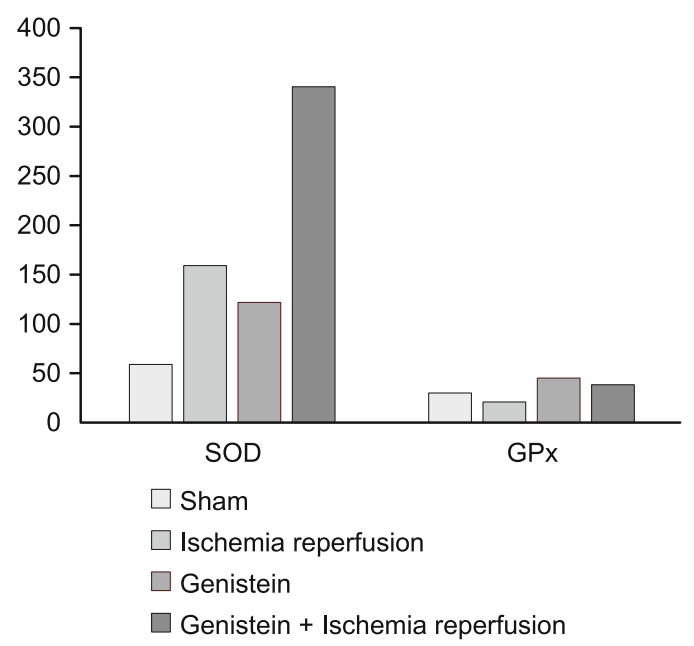

Fig. 3. The comparison of the SOD and GPx levels of the liver tissues of the groups.

tween the genistein $\mathrm{I} / \mathrm{R}$ and the $\mathrm{I} / \mathrm{R}$ groups was not statistically significant (Fig. 2).

The mean SOD level of the genistein + I / R group was significantly higher than in the other groups $(\mathrm{p}<0.001)$ (Fig. 3).

The mean GPx level was highest in the genistein group and the lowest in the I/R group. Moreover, the mean GPx level of the genistein $+\mathrm{I} / \mathrm{R}$ group was significantly higher than that of I/R group $(\mathrm{p}<0.001)$.

Histopathological evaluations including cholestasis, hydropic swelling, granular degeneration, microvesicular vacuolization, focal necrosis, cord irregularity, inflammation in the portal area, fibrosis and hyperemia in the sinusoid were performed. In the sham and genistein groups, normal histopathological appearance was observed in all tissue sections (Suzuki score: 0) (Fig. 4). I/R group revealed sinusoidal dilatation, hepatic vein congestion, hydropic degeneration and subcapsular necrosis (Fig. 5). Four rats in the I/R group had mild (Suzuki score 2), and four rats had

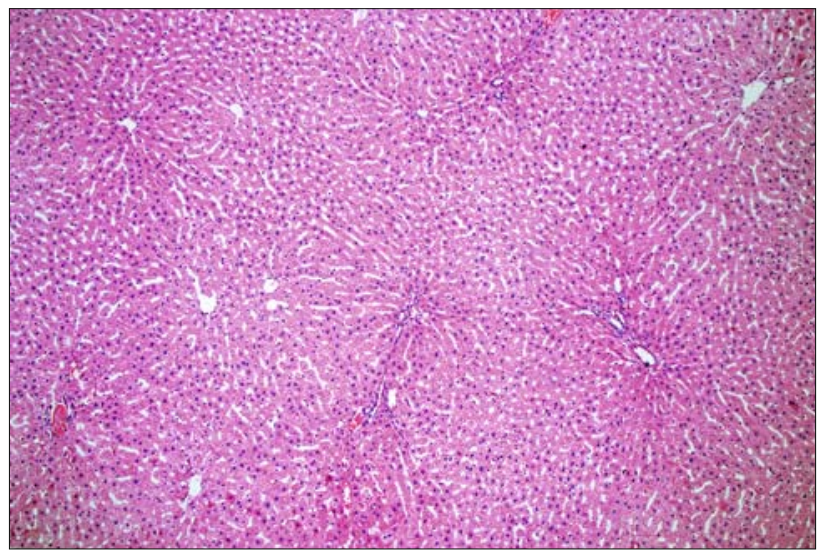

Fig. 4. Histopathological examination of the sham group demonstrates normal appearance of the hepatocytes (HE, X100).

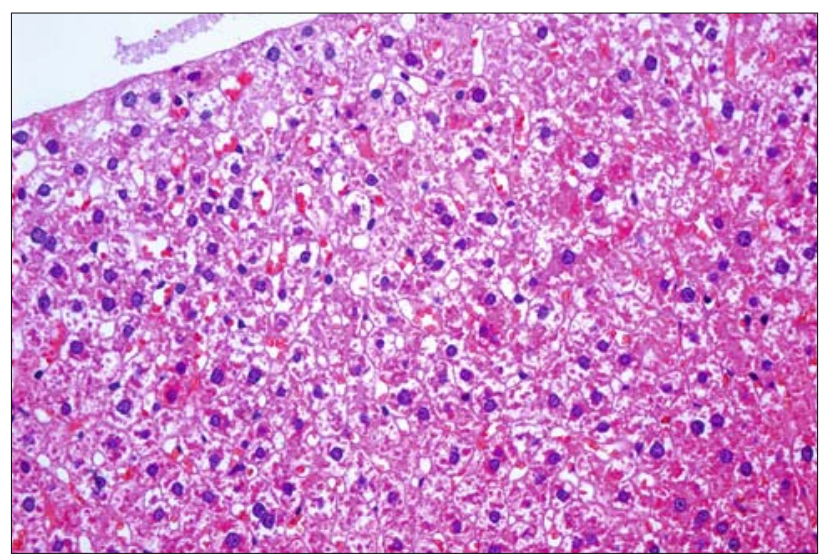

Fig. 5. The congestion and subcapsular necrosis in the I/R group (HE, $\mathrm{x} 400$ ).

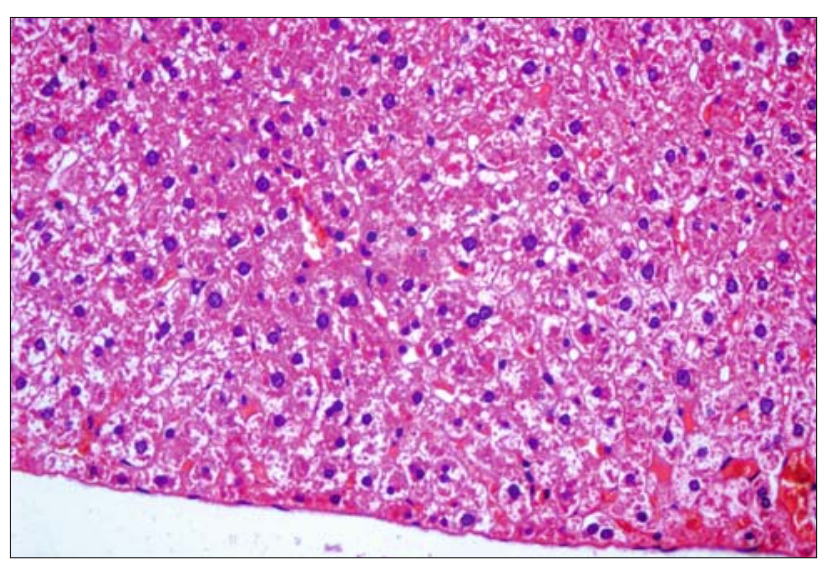

Fig. 6. Subcapsular necrosis observed in three rats in Genistein $+I / R$ group (HE, X400).

moderate hepatocyte injury (Suzuki score: 3 ). In genistein $+\mathrm{I} / \mathrm{R}$ group; one rat had normal histopathological appearance, three rats had minimal hepatocyte injury (Suzuki score 1), one rat had moderate hepatocyte injury (Suzuki score 2) (Fig. 6). The other three rats in the genistein $+\mathrm{I} / \mathrm{R}$ group had marked vacuolization 
Tab. 2. The Suzuki scores of liver tissue samples of the groups, $\mathbf{n}(\%)$.

\begin{tabular}{|c|c|c|c|c|c|}
\hline Suzuki score & Sham & Ischemia Reperfusion & Genistein & Genistein+Ischemia Reperfusion & $\mathrm{p}$ \\
\hline 0 & $8(100.0)$ & - & $8(100.0)$ & $1(12.5)$ & $<0.001$ \\
\hline 1 & - & - & - & $3(37.5)$ & \\
\hline 2 & - & $4(50.0)$ & - & $1(12.5)$ & \\
\hline 3 & - & $4(50.0)$ & - & $3(37.5)$ & \\
\hline 4 & - & - & - & - & \\
\hline
\end{tabular}

and congestion, and this was consistent with moderate hepatocyte injury (Suzuki score: 3 ). The comparison of Suzuki scores of the groups revealed a statistically significant difference between $\mathrm{I} / \mathrm{R}$ and Genistein + I/R groups $(\mathrm{p}<0.001)($ Tab. 2).

\section{Discussion}

Hepatic ischemia-reperfusion injury is a critical status that is frequently encountered in clinical disorders such as liver transplantation, liver resection, trauma, hypovolemic shock, and sepsis. Although the experimental studies on hepatic I/R injury have provided useful information about its pathogenesis, it is still an important cause of morbidity and mortality in liver surgery.

We can describe the pathophysiology of the ischemia-reperfusion injury as a series of interrelated cellular and humoral events. Reduction of cellular oxidative phosphorylation leads to a decrease in adenosine triphosphate (ATP) production and consequently to inhibition of Na-K ATPase pump in the cell membrane. The intracellular and extracellular balance of the ions, such as $\mathrm{H}^{+}, \mathrm{Na}^{+}$, and $\mathrm{Ca}^{2+}$ is impaired.

Subsequently, calcium is released from intracellular stores. The increased cytoplasmic calcium activates the various phospholipases, the membrane and membrane proteins that initiate membrane damage, the activated ATPases that accelerate ATP loss, and the endonucleases that break down genetic material (13). Ischemia-related ATP reduction leads to the accumulation of purine metabolites such as xanthine and hypoxanthine in the tissue and the conversion of xanthine dehydrogenase to xanthine oxidase. Xanthine oxidase converts hypoxanthine to uric acid, and molecular oxygen is used as the electron receiver for this reaction (14). Consequently, when oxygen is reperfused, excess accumulation of hypoxanthine induced by xanthine oxidase results in the formation of toxic free oxygen radicals (15).

The antioxidant defense system protects the organism by the removal or neutralization of the free oxygen radicals, preventing the formation of free radicals, and by improving the tissue damage (16). Numerous experimental studies are based on the hypothesis that the hepatic I/R injury can be minimized by exogenous antioxidant supply due to their potential inhibitory effect on the generation of the free oxygen radicals by antioxidant agents.

Genistein that was used in this study has well-known antioxidant properties and is a natural compound from the class of isoflavonoids found in high concentrations in legumes and soybeans. The present literature lacks the studies on the hepatic I/R model that used genistein, except a congress abstract presented by Yamamoto et al (17).

In the present study, the indicators of hepatocellular damage including the serum ALT, AST, and LDH levels were significantly decreased in the genistein $+\mathrm{I} / \mathrm{R}$ group compared to $\mathrm{I} / \mathrm{R}$ group. These data show that genistein has a protective effect on hepatic $\mathrm{I} / \mathrm{R}$ damage in liver tissue. Our study has similar results in terms of ALT, AST, and LDH parameters compared to previous experimental models in the literature. Various agents such as allopurinol, fish oil, melatonin, minocycline, rosiglitazone with antioxidant activity have been shown to provide a significant reduction in serum ALT, AST, LDH levels in hepatic I/R injury $(18,19,20,21)$.

TNF- $\alpha$ is one of the earliest released cytokines associated with reperfusion in hepatic I/R injury. Genistein provides inhibition of $\mathrm{NF}-\kappa \mathrm{B}$ activity and TNF- $\alpha$ release from macrophages by inhibiting the tyrosine-specific protein kinase activity of the epidermal growth factor receptor (22). In the present study, we have shown that genistein shows anti-inflammatory activity by decreasing serum TNF- $\alpha$ levels in hepatic ischemia-reperfusion injury.

Interleukin-6 that is released from endothelial and Kupffer cells plays an important role in the proliferative capacity of hepatocytes in liver regeneration. In a study with total flavonoids extracted from Rosa laevigata plant, it has been shown that flavonoids cause a significant reduction in the level of IL-6-related genes in the hepatic I/R injury (23). Similarly, it has been reported that genistein inhibits oxidative damage by decreasing IL-6 levels in rats with non-alcoholic steatohepatitis $(24,25)$. In contrast to the results of the literature, the mean IL- 6 was higher in the genistein + I/R group than in the other three groups, but no significant difference was found compared to I/R group. Similar to our study, an increase in IL-6 level was found in a study that used an ischemic preconditioning model (26). This result was not surprising because of the pro-mitogenic and anti-apoptotic effect of IL-6, even known as the pleiotropic biological effect.

The two crucial enzymes of the antioxidant defense system, SOD and GPx, which stop and neutralize the production of free oxygen radicals, are well known to play a protective role in ischemia-reperfusion injury by reducing oxidative stress. In an experimental rat study, genistein has been shown to increase the level of catalase in the small intestine, liver and kidney, and the concentration of SOD and GPx in the skin (27). In another study, it has been reported that genistein plays a protective role against the oxidative stress on endothelial cells by inducing Nrfl activation, one of the transcription factors that play a role in the reaction to oxidative stress (28). In the present study, the mean SOD and GPx levels were significantly higher in the genistein $+\mathrm{I} / \mathrm{R}$ group than the I/R group. Besides, the mean SOD and GPx levels of the genistein group were higher than those of the sham group. These results indicate that genistein has a favorable effect on the inhibition of hepatic oxidative stress.

In the present study, the comparison of the Suzuki scores of the groups for histopathological evaluation showed a significant 
difference between the genistein $+I / R$ and the I/R groups. This suggests that genistein has a cytoprotective effect on hepatocytes. We think that this cytoprotective effect of genistein is provided by inhibiting the excretion of hepatic enzymes and inducing the antioxidant defense system.

\section{Conclusion}

In conclusion, we suggest that genistein, with its antioxidant and anti-inflammatory properties, might cause favorable outcomes of hepatic surgery because of its protective effects on hepatic ischemia-reperfusion injury. These protective effects of the genistein have been significantly supported by the biochemical and histopathological findings of the present study. However, the effects of genistein need to be supplemented with further studies with larger numbers, so that it can be used to prevent hepatic ischemia-reperfusion injury in the clinical practice.

\section{Learning points}

- The indicators of hepatocellular damage including the serum ALT, AST, and LDH levels were significantly decreased in the genistein $+\mathrm{I} / \mathrm{R}$ group.

- Genistein shows anti-inflammatory activity by decreasing serum TNF- $\alpha$ levels in hepatic ischemia-reperfusion injury.

- The mean SOD and GPx levels were significantly higher in the genistein $+\mathrm{I} / \mathrm{R}$ group than the I/R group.

- Genistein has protective effects on hepatic ischemia-reperfusion injury.

\section{References}

1. Homer-Vanniasinkam S, Crinnion JN, Gough MJ. Post-ischemic organ dysfunction: a review. Eur J Vasc Endovasc Surg 1997; 14: 195-203.

2. Monsinjon T, Richard V, Fontaine M. Complement and its implications in cardiac ischemia/reperfusion: strategies to inhibit complement. Fundam Clin Pharmacol 2001; 15 (5): 293-306.

3. Teoh NC, Farrell GC. Hepatic ischemia reperfusion injury: Pathogenic mechanisms and basis for hepatoprotection. J Gastroenterol Hepatol 2003; 18 (8): 891-902.

4. Qian T, Nieminen AL, Herman B. Mitochondrial permeability transition in $\mathrm{pH}$ dependent reperfusion injury to rat hepatocytes. Am J Physiol 1997; 273: 1783-1792.

5. Camargo CA Jr, Madden JF, Gao W et al. Interleukin-6 protects against warm ischemia/reperfusion injury and promotes hepatocyte proliferation in the rodent. Hepatology 1997; 26: 1513-1520.

6. Baykara B, Tekmen I. Karaciğer İskemi Reperfüzyon Hasarı, Dokuz Eylül Üniversitesi, Tıp Fakültesi Dergisi. 2005; Cilt 19, Sayı 3; 185-194.

7. Hou Z, Yanaga K, Kamohara Y et al. A New Suppressive Agent Against İterleukin-1b And Tumor Necrosis Factor-A Enhances Liver Regeneration After Partial Hepatectomy in rats. Hepatol Res 2003; 26 (1): 40-46.

8. Polkowski K, Mazurek AP. Biological properties of genistein. A review of in vitro and in vivo data. Acta Pol Pharm 2000; 57 (2): 135-155

9. Li WF, Yang K, Zhu P et al. Genistein Ameliorates Ischemia/Reperfusion-Induced Renal Injury in a SIRT1-Dependent Manner. Nutrients 2017; 9 (4). doi: 10.3390/nu9040403.
10. Wang S, Wei H, Cai $M$ et al. Genistein attenuates brain damage induced by transient cerebral ischemia through up-regulation of ERK activity in ovariectomized mice. Int J Biol Sci 2014; 10 (4): 457-65.

11. Hayashi A, Weinberger AW, Kim HC et al. Genistein, a protein tyrosine kinase inhibitor, ameliorates retinal degeneration after ischemia-reperfusion injury in rat. Invest Ophthalmol Vis Sci 1997; 38 (6): 1193-1202.

12. Suzuki S, Nakamura S, Sakaguchi T et al. Alteration of reticuloendothelial phagocytic function and tumor necrosis factor-alpha production after total hepatic ischemia. Transplantation 1997; 64: 821-827.

13. Kumar V, Abbas A, Fausto N, Mitchell R. Robbins Basic Pathology. Philadelphia: Saunders Elsevier: 2007; 13-19.

14. Parks DA, Williams TK, Beckman JS. Conversion of xanthine dehydrogenase to oxidase in ischemic rat intestine: a reevaluation. Am J Physiol 1988; 254 (1): 768-774.

15. Toyokuni S. Reactive oxygen species-induced molecular damage and its application in pathology. Pathol Int 1999; 49: 91-102.

16. Girn HR, Ahilathirunayagam S, Mavor AI et al. Reperfusion Syndrome: Cellular mechanisms of microvascular dysfunction and potential therapeutic strategies. Vasc Endovascular Surg 2007; 41: 177-193.

17. Yamamoto S, Shimizu K, Oonishi I et al. Genistein supresses cellular injury following hepatic ishemia/reperfusion. Transplant Proc 1996; 28: 1111-1115.

18. Messiha BA, Abo-Youssef AM. Protective effects of fish oil, allopurinol and verapamil on hepatic ischemia-reperfusion injury in rats. J Nat Sci Biol Med 2015; 6: 351-355.

19. Deng WS, Xu Q, Liu YE et al. Effects of melatonin on liver function and lipid peroxidation in a rat model of hepatic ischemia reperfusion injury. Exp Ther Med 2016; 11: 1955-1960.

20. Yınıng L, Tao L, Hazhı Q, Fang Yuan. Minocycline protects against hepatic ischemia/reperfusion injury in a rat model. Biomed Rep 2015; 3 (1): 19-24.

21. Chen J, Liu H, Zhang $X$ et al. Protective effects of rosiglitazone on hepatic ischemia reperfusion injury in rats. J Central South Univ 2018; 43 (7): 732-737.

22. Gottstein N, Ewins BA, Eccleston $\mathbf{C}$ et al. Effect of genistein and daidzein on platelet aggregation and monocyte and endothelial function. Br J Nutr 2003; 89: 607-616.

23. Tao X, Sun X, Xu L et al. Total Flavonoids from Rosa laevigata Michx Fruit Ameliorates Hepatic Ischemia/Reperfusion Injury through Inhibition of Oxidative Stress and Inflammation in Rats. Nutrients 2016; 8 (7).

24. Ji G, Yang Q, Hao J et al. Anti-inflammatory effect of genistein on non-alcoholic steatohepatitis rats induced by high fat diet and its potential mechanisms. Int Immunopharmacol 2011; 11 (6): 762-768.

25. Mohamed Salih S, Nallasamy P, Muniyandi P et al. Genistein improves liver function and attenuates non-alcoholic fatty liver disease in a rat model of insulin resistance. J Diabetes 2009; 1 (4): 278-287.

26. Kannerup AS, Gronbaek H, Fuch-Jensen $P$ et al. Cytokine changes during warm ischemia and reperfusion of the pig liver with or without preconditioning. Eur Surg Res 2009; 42: 216-222.

27. Cai Q, Wei H. Effect of dietary genistein on antioxidant enzyme activities in SENCAR mice. Nutr Cancer 1996; 25 (1): 1-7.

28. Hernandez-Montes E, Pollard SE, Vauzour D et al. Activation of glutathione peroxidase via Nrf1 mediates genistein's protection against oxidative endothelial cell injury. Biochem Biophys Res Commun 2006; 346 (3): 851-859.

Received May 1, 2019. Accepted June 3, 2019. 